\title{
On a Hilbert-Type Integral Inequality with a Combination Kernel and Applications
}

BICHENG YANG

Department of Mathematics, Guangdong Education Institute, Guangzhou, Guangdong 510303, P. R. China

e-mail : bcyang@pub.guangzhou.gd.cn

ABSTRACT. By introducing some parameters and using the way of weight function and the technic of real analysis and complex analysis, a new Hilbert-type integral inequality with a best constant factor and a combination kernel involving two mean values is given, which is an extension of Hilbert's integral inequality. As applications, the equivalent form and the reverse forms are considered.

\section{Introduction}

If $0<\int_{0}^{\infty} f^{2}(x) d x<\infty$ and $0<\int_{0}^{\infty} g^{2}(x) d x<\infty$, then we have[1]:

$$
\begin{aligned}
& \int_{0}^{\infty} \int_{0}^{\infty} \frac{f(x) g(y)}{x+y} d x d y<\pi\left(\int_{0}^{\infty} f^{2}(x) d x \int_{0}^{\infty} g^{2}(x) d x\right)^{\frac{1}{2}} \\
& \int_{0}^{\infty} \int_{0}^{\infty} \frac{f(x) g(y)}{\max \{x, y\}} d x d y<4\left(\int_{0}^{\infty} f^{2}(x) d x \int_{0}^{\infty} g^{2}(x) d x\right)^{\frac{1}{2}}
\end{aligned}
$$

where the constant factor $\pi$ and 4 are all the best possible. We call (1) Hilbert's integral inequality. Both (1) and (2) are important in analysis and its applications[1,2]. In recent years, by using the way of weight function, a number of extensions of (1) and (2) were given by Yang et al. [3, 4]. In 2006, Li et al. [5] gave a new inequality with a combination kernel as follows:

$$
\int_{0}^{\infty} \int_{0}^{\infty} \frac{f(x) g(y)}{x+y+\max \{x, y\}} d x d y<c\left(\int_{0}^{\infty} f^{2}(x) d x \int_{0}^{\infty} g^{2}(x) d x\right)^{\frac{1}{2}},
$$

where the constant factor $c=2 \sqrt{2} \arctan \frac{1}{\sqrt{2}}$ is the best possible. In 2007, Xie [6] gave a best extension of (3) and Guo et al. [7, 8] gave a similar form of (3) as follows:

$$
\int_{0}^{\infty} \int_{0}^{\infty} \frac{f(x) g(y) d x d y}{x+y+\min \{x, y\}}<\widetilde{c}\left(\int_{0}^{\infty} f^{2}(x) d x \int_{0}^{\infty} g^{2}(x) d x\right)^{\frac{1}{2}}
$$

Received July 7, 2009; accepted August 21, 2009.

2000 Mathematics Subject Classification: 26D15.

Key words and phrases: Hilbert-type integral inequality, weight function, parameter. 
where the constant factor $\widetilde{c}=2 \sqrt{2} \arctan \sqrt{2}$ is the best possible.

We know the following mean values inequalities:

$$
\max \{x, y\} \geq \frac{x+y}{2} \geq \sqrt{x y} \geq \frac{2}{x^{-1}+y^{-1}} \geq \min \{x, y\}(x, y>0) .
$$

It means that $x+y-4\left(x^{-1}+y^{-1}\right)^{-1} \geq 0$.

In this paper, by introducing some parameters and using the way of weight function and the technic of real analysis and complex analysis, we give a new Hilberttype integral inequality with a combination kernel as $\frac{1}{x^{\lambda}+y^{\lambda}+A\left(x^{-\lambda}+y^{-\lambda}\right)^{-1}}(\lambda>$ $0, A>-4)$, which is an extension of (1). As applications, the equivalent form and the reverse forms are obtained.

\section{Some Lemmas}

Lemma 1. If $\lambda>0, A>-4$, then we have

$$
\begin{gathered}
\widetilde{K}_{\lambda}(A):=\int_{0}^{\infty} \frac{u^{\frac{\lambda}{2}-1} d u}{u^{\lambda}+1+A\left(u^{-\lambda}+1\right)^{-1}}=\frac{2 \pi}{\lambda \sqrt{A+4}} ; \\
\varpi_{\lambda}(y):=\int_{0}^{\infty} \frac{y^{\frac{\lambda}{2}} x^{\frac{\lambda}{2}-1} d x}{x^{\lambda}+y^{\lambda}+A\left(x^{-\lambda}+y^{-\lambda}\right)^{-1}}=\widetilde{K}_{\lambda}(A), \quad(y \in(0, \infty)) .
\end{gathered}
$$

Proof. Setting $v=u^{\lambda / 2}$, by calculation, we find

$$
\widetilde{K}_{\lambda}(A)=\frac{1}{\lambda} \int_{-\infty}^{\infty} \frac{v^{2}+1}{(v+1)^{2}+A v^{2}} d v
$$

Since for $A>-4$, it follows

$$
\begin{aligned}
(v+1)^{2}+A v^{2} & =\left(v^{2}+1-\sqrt{-A} v\right)\left(v^{2}+1+\sqrt{-A} v\right) \\
& =\left(v-v_{1}\right)\left(v-v_{2}\right)\left(v-v_{3}\right)\left(v-v_{4}\right),
\end{aligned}
$$

where $v_{1}=\frac{1}{2}(\sqrt{-A}+\sqrt{A+4} i), v_{2}=\frac{1}{2}(\sqrt{-A}-\sqrt{A+4} i), v_{3}=\frac{1}{2}(-\sqrt{-A}+$ $\sqrt{A+4} i), v_{4}=\frac{1}{2}(-\sqrt{-A}-\sqrt{A+4} i)$. Obviously, we can find that Im $v_{1}>0$ and $\operatorname{Im} v_{3}>0$, for $-4<A \leq 0$ and for $A>0$. Setting a complex function as $f(z)=\frac{z^{2}+1}{\left(z-v_{1}\right)\left(z-v_{2}\right)\left(z-v_{3}\right)\left(z-v_{4}\right)}$, in view of the theorem of obtaining real integral by using residue[9], (a) if $A \neq 0$, then $v_{1} \neq v_{3}$ and

$$
\begin{aligned}
& \underset{z=v_{1}}{\operatorname{Res}} f(z)=\frac{v_{1}^{2}+1}{\left(v_{1}-v_{2}\right)\left(v_{1}-v_{3}\right)\left(v_{1}-v_{4}\right)}=\frac{1}{2 \sqrt{A+4} i} ; \\
& \underset{z=v_{3}}{\operatorname{Res}} f(z)=\frac{v_{3}^{2}+1}{\left(v_{3}-v_{1}\right)\left(v_{3}-v_{2}\right)\left(v_{3}-v_{4}\right)}=\frac{1}{2 \sqrt{A+4} i} ;
\end{aligned}
$$




$$
k_{\lambda}(A)=\frac{1}{\lambda} \int_{-\infty}^{\infty} f(v) d v=\frac{2 \pi i}{\lambda}\left(\underset{z=v_{1}}{\operatorname{Res}} f(z)+\underset{z=v_{3}}{\operatorname{Res}} f(z)\right)=\frac{2 \pi}{\lambda \sqrt{A+4}}
$$

(b) if $A=0$, then $v_{1}=v_{3}=i, v_{2}=v_{4}=-i$ and

$$
\begin{aligned}
\underset{z=i}{\operatorname{Res}} f(z) & =\left[\frac{z^{2}+1}{(z+i)^{2}}\right]_{z=i}^{\prime}=\frac{1}{2 i} \\
k_{\lambda}(A) & =\frac{1}{\lambda} \int_{-\infty}^{\infty} f(v) d v=\frac{2 \pi i}{\lambda} \operatorname{Res}_{z=i} f(z)=\frac{\pi}{\lambda}
\end{aligned}
$$

Hence (6) is valid. Setting $u=x / y$, we obtain (7). The lemma is proved.

Lemma 2. Assume that $p>0(p \neq 1),|q|>0, \lambda>0, A>-4$ and $0<\varepsilon<$ $\frac{\lambda}{2} \min \{p,|q|\}$. Then for $\varepsilon \rightarrow 0^{+}$, we have

(8) $\quad \int_{0}^{1} \frac{u^{\frac{\lambda}{2}+\frac{\varepsilon}{q}-1} d u}{u^{\lambda}+1+A\left(u^{-\lambda}+1\right)^{-1}}=\int_{0}^{1} \frac{u^{\frac{\lambda}{2}-1} d u}{u^{\lambda}+1+A\left(u^{-\lambda}+1\right)^{-1}}+o_{1}(1)$;

(9) $\int_{1}^{\infty} \frac{u^{\frac{\lambda}{2}-\frac{\varepsilon}{p}-1} d u}{u^{\lambda}+1+A\left(u^{-\lambda}+1\right)^{-1}}=\int_{1}^{\infty} \frac{u^{\frac{\lambda}{2}-1} d u}{u^{\lambda}+1+A\left(u^{-\lambda}+1\right)^{-1}}+o_{2}(1)$;

(10) $\int_{0}^{1} \frac{u^{\frac{\lambda}{2}-\frac{\varepsilon}{p}-1} d u}{u^{\lambda}+1+A\left(u^{-\lambda}+1\right)^{-1}}=\int_{0}^{1} \frac{u^{\frac{\lambda}{2}-1} d u}{u^{\lambda}+1+A\left(u^{-\lambda}+1\right)^{-1}}+o_{3}(1)$.

Proof. (a) If $-4<A<0$, setting $\eta_{A}=\frac{1}{4}(A+4)>0$, then we obtain

$$
\begin{aligned}
u^{\lambda}+1+A\left(u^{-\lambda}+1\right)^{-1} & =\eta_{A}\left(u^{\lambda}+1\right)+\left(1-\eta_{A}\right)\left(u^{\lambda}+1\right)+\frac{A u^{\lambda}}{1+u^{\lambda}} \\
& \geq \eta_{A}\left(u^{\lambda}+1\right)+\frac{4\left(1-\eta_{A}\right) u^{\lambda}}{1+u^{\lambda}}+\frac{A u^{\lambda}}{1+u^{\lambda}} \\
& =\eta_{A}\left(u^{\lambda}+1\right) \geq\left\{\begin{array}{l}
\eta_{A}, u \in(0,1] \\
\eta_{A} u^{\lambda}, u \in(1, \infty)
\end{array}\right.
\end{aligned}
$$


(b) if $A \geq 0$, setting $\eta_{A}=1$, then we still have (11). For $\varepsilon \rightarrow 0^{+}$, we find

$$
\begin{aligned}
0 & <\int_{0}^{1} \frac{u^{\frac{\lambda}{2}-1} d u}{u^{\lambda}+1+A\left(u^{-\lambda}+1\right)^{-1}}-\int_{0}^{1} \frac{u^{\frac{\lambda}{2}+\frac{\varepsilon}{q}-1} d u}{u^{\lambda}+1+A\left(u^{-\lambda}+1\right)^{-1}} \\
& \leq \frac{1}{\eta_{A}} \int_{0}^{1} u^{\frac{\lambda}{2}-1}\left(1-u^{\frac{\varepsilon}{q}}\right) d u \leq \frac{1}{\eta_{A}}\left(\frac{2}{\lambda}-\frac{1}{\frac{\lambda}{2}+\frac{\varepsilon}{q}}\right) \rightarrow 0 ; \\
0 & <\int_{1}^{\infty} \frac{u^{\frac{\lambda}{2}-1} d u}{u^{\lambda}+1+A\left(u^{-\lambda}+1\right)^{-1}}-\int_{1}^{\infty} \frac{u^{\frac{\lambda}{2}-\frac{\varepsilon}{p}-1} d u}{u^{\lambda}+1+A\left(u^{-\lambda}+1\right)^{-1}} \\
& \leq \frac{1}{\eta_{A}} \int_{1}^{\infty} \frac{u^{\frac{\lambda}{2}-1}\left(1-u^{-\frac{\varepsilon}{p}}\right) d u}{u^{\lambda}}=\frac{1}{\eta_{A}}\left(\frac{2}{\lambda}-\frac{1}{\frac{\lambda}{2}+\frac{\varepsilon}{p}}\right) \rightarrow 0 ; \\
0 & <\int_{0}^{1} \frac{u^{\frac{\lambda}{2}-\frac{\varepsilon}{p}-1} d u}{u^{\lambda}+1+A\left(u^{-\lambda}+1\right)^{-1}}-\int_{0}^{1} \frac{u^{\frac{\lambda}{2}-1} d u}{u^{\lambda}+1+A\left(u^{-\lambda}+1\right)^{-1}} \\
& \leq \frac{1}{\eta_{A}} \int_{0}^{1} u^{\frac{\lambda}{2}-1}\left(u^{-\frac{\varepsilon}{p}}-1\right) d u \leq \frac{1}{\eta_{A}}\left(\frac{1}{\frac{\lambda}{2}-\frac{\varepsilon}{p}}-\frac{2}{\lambda}\right) \rightarrow 0 .
\end{aligned}
$$

Hence Expressions (8), (9) and (10) are valid. The lemma is proved.

\section{Main results and applications}

Theorem 1. If $\lambda, p>0(p \neq 1), \frac{1}{p}+\frac{1}{q}=1, A>-4, \widetilde{K}_{\lambda}(A)$ is expressed by (6), $\phi_{r}(x)=x^{r\left(1-\frac{\lambda}{2}\right)-1}(r=p, q), f, g \geq 0,0<\|f\|_{p, \phi_{p}}:=\left\{\int_{0}^{\infty} \phi_{p}(x) f^{p}(x) d x\right\}^{\frac{1}{p}}<\infty$ and $0<\|g\|_{q, \phi_{q}}=\left\{\int_{0}^{\infty} \phi_{q}(x) g^{q}(x) d x\right\}^{\frac{1}{q}}<\infty$, then

(a) for $p>1$, we have the following equivalent inequalities:

$$
I_{\lambda}:=\int_{0}^{\infty} y^{\frac{p \lambda}{2}-1}\left[\int_{0}^{\infty} \frac{f(x) d x}{x^{\lambda}+y^{\lambda}+A\left(x^{-\lambda}+y^{-\lambda}\right)^{-1}}\right]^{p} d y<\widetilde{K}_{\lambda}^{p}(A)\|f\|_{p, \phi_{p}}^{p}
$$

$$
J_{\lambda}:=\int_{0}^{\infty} \int_{0}^{\infty} \frac{f(x) g(y) d x d y}{x^{\lambda}+y^{\lambda}+A\left(x^{-\lambda}+y^{-\lambda}\right)^{-1}}<\widetilde{K}_{\lambda}(A)\|f\|_{p, \phi_{p}}\|g\|_{q, \phi_{q}}
$$

(b) for $0<p<1$, we have the equivalent reverse forms of (12) and (13).

Proof. By Hölder's inequality[10] and (7), for $y \in(0, \infty)$, we obtain

$$
\begin{aligned}
& {\left[\int_{0}^{\infty} \frac{f(x) d x}{x^{\lambda}+y^{\lambda}+A\left(x^{-\lambda}+y^{-\lambda}\right)^{-1}}\right]^{p} } \\
= & \left\{\int_{0}^{\infty} \frac{1}{x^{\lambda}+y^{\lambda}+A\left(x^{-\lambda}+y^{-\lambda}\right)^{-1}}\left[\frac{x^{\left(1-\frac{\lambda}{2}\right) / q}}{y^{\left(1-\frac{\lambda}{2}\right) / p}} f(x)\right]\left[\frac{y^{\left(1-\frac{\lambda}{2}\right) / p}}{x^{\left(1-\frac{\lambda}{2}\right) / q}}\right] d x\right\}^{p}
\end{aligned}
$$




$$
\begin{aligned}
& \leq\left[\int_{0}^{\infty} \frac{x^{\left(1-\frac{\lambda}{2}\right)(p-1)} y^{\frac{\lambda}{2}-1} f^{p}(x) d x}{x^{\lambda}+y^{\lambda}+A\left(x^{-\lambda}+y^{-\lambda}\right)^{-1}}\right]\left[\int_{0}^{\infty} \frac{y^{\left(1-\frac{\lambda}{2}\right)(q-1)} x^{\frac{\lambda}{2}-1} d x}{x^{\lambda}+y^{\lambda}+A\left(x^{-\lambda}+y^{-\lambda}\right)^{-1}}\right]^{p-1} \\
& =\widetilde{K}_{\lambda}^{p-1}(A) y^{1-\frac{p \lambda}{2}} \int_{0}^{\infty} \frac{x^{\left(1-\frac{\lambda}{2}\right)(p-1)} y^{\frac{\lambda}{2}-1}}{x^{\lambda}+y^{\lambda}+A\left(x^{-\lambda}+y^{-\lambda}\right)^{-1}} f^{p}(x) d x
\end{aligned}
$$

By (14), in view of Fubini's theorem[11] and (7), it follows

$$
\begin{aligned}
I_{\lambda} & \leq \widetilde{K}_{\lambda}^{p-1}(A) \int_{0}^{\infty} \int_{0}^{\infty} \frac{x^{\left(1-\frac{\lambda}{2}\right)(p-1)} y^{\frac{\lambda}{2}-1} f^{p}(x)}{x^{\lambda}+y^{\lambda}+A\left(x^{-\lambda}+y^{-\lambda}\right)^{-1}} d x d y \\
& =\widetilde{K}_{\lambda}^{p-1}(A) \int_{0}^{\infty} \varpi(x) \phi_{p}(x) f^{p}(x) d x=\widetilde{K}_{\lambda}^{p}(A)\|f\|_{p, \phi_{p}}^{p} .
\end{aligned}
$$

If there exists a $y \in(0, \infty)$, such that (14 ) takes the form of equality, then[10] there exists constants $a$ and $b$, such that they are not all zero and $x^{1-\frac{\lambda}{2}(p-1)} y^{\frac{\lambda}{2}-1} f^{p}(x)=y^{\left(1-\frac{\lambda}{2}\right)(q-1)} x^{\frac{\lambda}{2}-1}$ a.e. in $(0, \infty)$. It means that $a x^{p\left(1-\frac{\lambda}{2}\right)} f^{p}(x)=b y^{q\left(1-\frac{\lambda}{2}\right)}$ a.e. in $(0, \infty)$. We affirm that $a \neq 0$, otherwise $b=a=0$. Hence it follows $x^{p\left(1-\frac{\lambda}{2}\right)-1} f^{p}(x)=\left[b y^{q\left(1-\frac{\lambda}{2}\right)}\right] /(a x)$ a.e. in $(0, \infty)$, which contradicts the fact that $0<\|f\|_{p, \phi_{p}}<\infty$. Then (14) keeps the form of strict inequality; so does (15). And (12) is valid.

By Hölder's inequality[10], we find

$$
J_{\lambda}=\int_{0}^{\infty}\left[\int_{0}^{\infty} \frac{y^{\frac{-1}{p}+\frac{\lambda}{2}} f(x) d x}{x^{\lambda}+y^{\lambda}+A\left(x^{-\lambda}+y^{-\lambda}\right)^{-1}}\right]\left[y^{\frac{1}{p}-\frac{\lambda}{2}} g(y)\right] d y \leq I_{\lambda}^{\frac{1}{p}}\|g\|_{q, \phi_{q}}
$$

In view of (12), we have (13).

On the other-hand, suppose that (13) is valid. There exists $n_{0} \in N$, such that for $n \geq n_{0}, 0<\int_{1 / n}^{n} \phi_{p}(x)[f(x)]_{n}^{p} d x<\infty$, where $[f(x)]_{n}=n$, for $f(x) \geq n ;[f(x)]_{n}=$ $f(x)$, for $f(x)<n$. For $n \geq n_{0}$, setting

$$
g_{n}(y):=y^{\frac{p \lambda}{2}-1}\left[\int_{1 / n}^{n} \frac{[f(x)]_{n} d x}{x^{\lambda}+y^{\lambda}+A\left(x^{-\lambda}+y^{-\lambda}\right)^{-1}}\right]^{p-1}, y \in(0, n],
$$

by (13), we find

$$
\text { (18) } \begin{aligned}
0 & <\int_{\frac{1}{n}}^{n} \phi_{q}(y) g_{n}^{q}(y) d y=\int_{\frac{1}{n}}^{n} y^{\frac{p \lambda}{2}-1}\left[\int_{\frac{1}{n}}^{n} \frac{[f(x)]_{n} d x}{x^{\lambda}+y^{\lambda}+A\left(x^{-\lambda}+y^{-\lambda}\right)^{-1}}\right]^{p} d y \\
& =\int_{1 / n}^{n} \int_{1 / n}^{n} \frac{[f(x)]_{n} g_{n}(y)}{x^{\lambda}+y^{\lambda}+A\left(x^{-\lambda}+y^{-\lambda}\right)^{-1}} d x d y \\
& <\widetilde{K}_{\lambda}(A)\left\{\int_{1 / n}^{n} \phi_{p}(x)[f(x)]_{n}^{p} d x\right\}^{\frac{1}{p}}\left\{\int_{1 / n}^{n} \phi_{q}(y) g_{n}^{q}(y) d y\right\}^{\frac{1}{q}}<\infty ;
\end{aligned}
$$




$$
\left\{\int_{1 / n}^{n} \phi_{q}(y) g_{n}^{q}(y) d y\right\}^{\frac{1}{p}}<\widetilde{K}_{\lambda}(A)\left\{\int_{0}^{\infty} \phi_{p}(x) f^{p}(x) d x\right\}^{\frac{1}{p}}<\infty .
$$

Hence $0<\int_{0}^{\infty} \phi_{q}(y) g_{\infty}^{q}(y) d y<\infty$, and then (18) and (19) are valid for $n \rightarrow \infty$ by using (13). Therefore we obtain (12), which is equivalent to (13).

(b) By the reverse Hölder's inequality and the same way, we can obtain the reverse forms of (12) and (16) and then deduce the reverse form of (13). Setting $g_{n}(y)$ as (17), by the reverse form of (13), we obtain the reverse forms of (18) and (19), and then deduce the reverse form of (13), which is equivalent to the reverse form of (12). The theorem is proved.

Theorem 2. As the assumption of Theorem 1, all the constant factors in (12), (13) and the reverse forms are the best possible.

Proof. For $0<\varepsilon<\frac{\lambda}{2} \min \{p,|q|\}$, we set $f_{\varepsilon}, g_{\varepsilon}$ as: $f_{\varepsilon}(x)=g_{\varepsilon}(x)=0$, for $x \in(0,1)$; $f_{\varepsilon}(x)=x^{\frac{\lambda}{2}-\frac{\varepsilon}{p}-1}, g_{\varepsilon}(x)=x^{\frac{\lambda}{2}-\frac{\varepsilon}{q}-1}$, for $x \in[1, \infty)$.

(a) For $p>1$, if there exists a constant $0<k \leq \widetilde{K}_{\lambda}(A)$, such that (13) is still valid as we replace $\widetilde{K}_{\lambda}(A)$ by $k$, then in particular, we have

$$
\begin{aligned}
k & =\varepsilon k\left\|f_{\varepsilon}\right\|_{p, \phi_{p}}\left\|g_{\varepsilon}\right\|_{q, \phi_{q}}>\varepsilon \int_{0}^{\infty} \int_{0}^{\infty} \frac{f_{\varepsilon}(x) g_{\varepsilon}(y) d x d y}{x^{\lambda}+y^{\lambda}+A\left(x^{-\lambda}+y^{-\lambda}\right)^{-1}} \\
& =\varepsilon \int_{1}^{\infty} x^{\frac{\lambda}{2}-\frac{\varepsilon}{p}-1}\left[\int_{1}^{\infty} \frac{y^{\frac{\lambda}{2}-\frac{\varepsilon}{q}-1} d y}{x^{\lambda}+y^{\lambda}+A\left(x^{-\lambda}+y^{-\lambda}\right)^{-1}}\right] d x .
\end{aligned}
$$

Setting $u=x / y$ in the above integral, by Fubini's theorem, we find

$$
\begin{aligned}
k & >\varepsilon \int_{1}^{\infty} x^{-\varepsilon-1}\left[\int_{0}^{x} \frac{u^{\frac{\lambda}{2}+\frac{\varepsilon}{q}-1}}{u^{\lambda}+1+A\left(u^{-\lambda}+1\right)^{-1}} d u\right] d x \\
& =\int_{0}^{1} \frac{u^{\frac{\lambda}{2}+\frac{\varepsilon}{q}-1} d u}{u^{\lambda}+1+A\left(u^{-\lambda}+1\right)^{-1}}+\int_{1}^{\infty} x^{-\varepsilon-1}\left[\int_{1}^{x} \frac{\varepsilon u^{\frac{\lambda}{2}+\frac{\varepsilon}{q}-1} d u}{u^{\lambda}+1+A\left(u^{-\lambda}+1\right)^{-1}}\right] d x \\
& =\int_{0}^{1} \frac{u^{\frac{\lambda}{2}+\frac{\varepsilon}{q}-1} d u}{u^{\lambda}+1+A\left(u^{-\lambda}+1\right)^{-1}}+\varepsilon \int_{1}^{\infty} \frac{\left(\int_{u}^{\infty} x^{-\varepsilon-1} d x\right) u^{\frac{\lambda}{2}+\frac{\varepsilon}{q}-1}}{u^{\lambda}+1+A\left(u^{-\lambda}+1\right)^{-1}} d u \\
& =\int_{0}^{1} \frac{u^{\frac{\lambda}{2}+\frac{\varepsilon}{q}-1} d u}{u^{\lambda}+1+A\left(u^{-\lambda}+1\right)^{-1}}+\int_{1}^{\infty} \frac{u^{\frac{\lambda}{2}-\frac{\varepsilon}{p}-1}}{u^{\lambda}+1+A\left(u^{-\lambda}+1\right)^{-1}} d u .
\end{aligned}
$$

For $\varepsilon \rightarrow 0^{+}$, in view of (8) and (9), we find $k \geq \widetilde{K}_{\lambda}(A)$. Hence $k=\widetilde{K}_{\lambda}(A)$ is the best constant factor of (13). If the constant factor in (12) is not the best possible, then by (16), we may get a contradiction that the constant factor in (13) is not the best possible. 
(b) For $0<p<1$, if there exists $K \geq \widetilde{K}_{\lambda}(A)$, such that the reverse form of (13) is valid as we replace $\widetilde{K}_{\lambda}(A)$ by $K$, then we have

$$
\begin{aligned}
K & =\varepsilon K\left\|f_{\varepsilon}\right\|_{p, \phi_{p}}\left\|g_{\varepsilon}\right\|_{q, \phi_{q}}<\int_{0}^{\infty} \int_{0}^{\infty} \frac{\varepsilon f_{\varepsilon}(x) g_{\varepsilon}(y) d x d y}{\max \left\{x^{\lambda}, y^{\lambda}\right\}+A\left(x^{-\lambda}+y^{-\lambda}\right)^{-1}} \\
& \leq \varepsilon \int_{1}^{\infty} y^{\frac{\lambda}{2}-\frac{\varepsilon}{q}-1}\left[\int_{0}^{\infty} \frac{x^{\frac{\lambda}{2}-\frac{\varepsilon}{p}-1} d x}{x^{\lambda}+y^{\lambda}+A\left(x^{-\lambda}+y^{-\lambda}\right)^{-1}}\right] d y \\
& =\int_{0}^{1} \frac{u^{\frac{\lambda}{2}-\frac{\varepsilon}{p}-1} d u}{u^{\lambda}+1+A\left(u^{-\lambda}+1\right)^{-1}}+\int_{1}^{\infty} \frac{u^{\frac{\lambda}{2}-\frac{\varepsilon}{p}-1} d u}{u^{\lambda}+1+A\left(u^{-\lambda}+1\right)^{-1}} \\
& \leq \int_{0}^{1} \frac{u^{\frac{\lambda}{2}-\frac{\varepsilon}{p}-1} d u}{u^{\lambda}+1+A\left(u^{-\lambda}+1\right)^{-1}}+\int_{1}^{\infty} \frac{u^{\frac{\lambda}{2}-1} d u}{u^{\lambda}+1+A\left(u^{-\lambda}+1\right)^{-1}} .
\end{aligned}
$$

For $\varepsilon \rightarrow 0^{+}$, in view of (10), we obtain $K \leq \widetilde{K}_{\lambda}(A)$. Hence $K=\widetilde{K}_{\lambda}(A)$ is the best constant factor of the reverse form of (13). If the constant factor in the reverse form of (12) is not the best possible, then by the reverse form of (16), we may get a contradiction that the constant factor in the reverse form of (13) is not the best possible. The theorem is proved.

Remark. (a) For $p=q=2, \lambda=1, A=0$ in (13), it deduces to (1). Hence inequality (13) is an extension of (1);

(b) for $p>1, A=-3, \lambda>0$ in (13), we obtain a new inequality with the best constant factor $\frac{2 \pi}{\lambda}$ as

$$
\int_{0}^{\infty} \int_{0}^{\infty} \frac{f(x) g(y) d x d y}{\max \left\{x^{\lambda}, y^{\lambda}\right\}-3\left(x^{-\lambda}+y^{-\lambda}\right)^{-1}}<\frac{2 \pi}{\lambda}\|f\|_{p, \phi_{p}}\|g\|_{q, \phi_{q}}
$$

and it follows that the revere form of $(20)$ with the same best constant factor for $0<p<1, \frac{1}{p}+\frac{1}{q}=1$ and $0<\|f\|_{p, \phi_{p}},\|g\|_{q, \phi_{q}}<\infty$ is valid.

\section{References}

[1] G. H. Hardy, J. E. Littlewood and G. Polya, Inequalities, Cambridge Univ. Press, Cambridge, 1952.

[2] D. S. Mintrinovic, J. E. Pecaric and A. M. Kink, Inequalities involving functions and their integrals and derivertives, Kluwer Academic Publishers, Boston, 1991.

[3] Bicheng Yang, On a generalization of the Hilbert's type inequality and its applications, Chinese Journal of Engineering Mathematics, 21, 5(2004), 821-824.

[4] Bicheng Yang, Ilko Brnetic, Mario Krnic and Josip Pecaric, Generalization of Hilbert and Hardy-Hilbert integral inequalities, Mathematical Inequalities and Applications, 8, 2(2005), 259-272. 
[5] Yongjin Li and Bing He, A new Hilbert-type integral inequality and the equivalent form, Internat. J. Math. \& Math. Soc., Vol.2006, Arc. ID 457378:1-6.

[6] Chune Xie, Best generalization of a new Hilbert-type inequality, Journal of Jinan University (Natural Science), 28, 1(2007), 24-27.

[7] Xiaokui Ge, New extension of a Hilbert-type integral inequality, Journal of Jinan University (Natural Science), 28, 5(2007), 447-450.

[8] Yongjin Li and Bing He, On inequalities of Hilbert's type, Bulletin of the Australian Mathematical Society, 76(2007), 1-13.

[9] Yuquang Zhong, Complex analysis, Higher Education Press, Beijin, 2003.

[10] Jichang Kuang, Applied Inequalities, Shangdong Science Press, Jinan, 2004.

[11] Jichang Kuang, Introduction to real analysis, Hunan Education Press, Changsha, 1996 